\title{
NAFLD and COVID-19: a Pooled Analysis
}

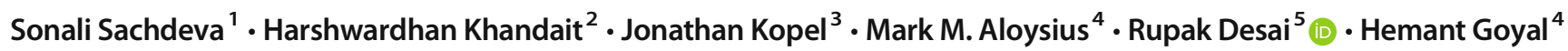

Accepted: 2 November 2020 / Published online: 6 November 2020

(C) Springer Nature Switzerland AG 2020

\begin{abstract}
The earliest evidence from China suggested that COVID-19 patients are even more vulnerable to succumbing from complications in the presence of a multimorbid status, including metabolic syndrome. Due to ongoing metabolic abnormalities, nonalcoholic fatty liver disease (NAFLD) appears to be a potential risk factor for contracting SARS-CoV-2 infection and developing related complications. This is because of the interplay of chronically active inflammatory pathways in NAFLD- and COVID-19associated acute cytokine storm. The risk of severe disease could also be attributed to compromised liver function as a result of NAFLD. We systematically reviewed current literature to ascertain the relationship between NAFLD and severe COVID-19, independent of obesity, which is considered the major factor risk factor for both NAFLD and COVID-19. We found that NAFLD is a predictor of severe COVID-19, even after adjusting for the presence of obesity (OR 2.358; 95\% CI: 1.902-2.923, $p<0.001$ ).
\end{abstract}

Keywords SARS-CoV-2 $\cdot$ COVID-19 $\cdot$ Coronavirus $\cdot$ NAFLD $\cdot$ MAFLD $\cdot$ Fatty liver

\section{Introduction}

Coronavirus disease 2019 (COVID-19) caused by Severe Acute Respiratory Syndrome Coronavirus-2 (SARS-CoV2) was declared a pandemic by the World Health Organization (WHO) in March 2020. Although it is primarily a respiratory illness, transaminemia associated with COVID-19 is associated with higher mortality [1]. The initial reports from liver biopsies of COVID-19 patients showed ballooning hepatocytes with mitotic cells and eosinophilic bodies, suggesting SARS-CoV-2 induces

This article is part of the Topical Collection on Covid-19

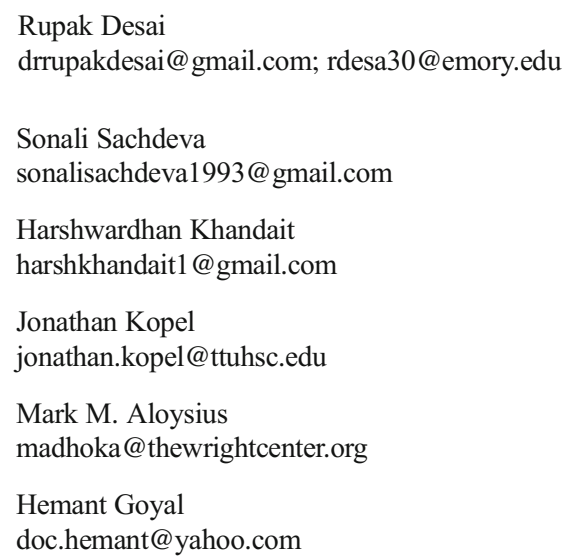

apoptosis in liver cells [1]. Other authors have proposed that the increased levels of inflammatory mediators, such as IL-1, IL-6, and IL-10 levels, may further contribute to damaging hepatocytes in COVID-19 patients [1]. Hepatic steatosis is a commonly observed finding in patients testing positive for SARS-CoV-2 [2]. The effect of fatty liver on the severity of COVID-19 illness has not yet been studied. We aim to systematically review published literature and evaluate the severity of COVID-19 in patients with fatty liver. Delhi, India

2 Department of Medicine, Government Medical College, Nagpur, India

3 Department of Medicine, Texas Tech University Health Sciences Center, Lubbock, TX, USA

4 The Wright Center for Graduate Medical Education, Scranton, PA, USA

5 Division of Cardiology, Atlanta VA Medical Center, 1670 Clairmont Rd, Decatur, GA 30033, USA 


\section{Methods}

A systematic literature search of PUBMED, Google Scholar, and SCOPUS databases was performed to identify studies reporting the association of fatty liver and COVID-19 through September 9, 2020. Search key terms employed were as follows: "NAFLD," "metabolic associated fatty liver disease (MAFLD)," "fatty liver," "COVID-19," and "SARS-COV2." The reference lists of these articles were also screened and hand-searched to identify further relevant studies. Included studies assessed the presence or absence of fatty liver by computed tomography $(\mathrm{CT})$ or abdominal ultrasound coupled with an internal expert consensus statement to diagnose NAFLD. The presence of positive SARS-CoV-2 RT-PCR diagnosed COVID-19 infection. Whenever available, we collected data on the risk COVID-19 and its severity in patients diagnosed with fatty liver. Severe COVID-19 was defined in most studies using guidelines laid down by the National Health Commission of China. Other outcomes included were hospitalization and ICU admission, as mentioned in Table 1. We included studies that reported OR for severe COVID-19 in patients with NAFLD/MAFLD after adjusting for obesity. Data on the odds ratio (OR) with $95 \%$ confidence interval (CI) were collected and pooled to compute the pooled odds ratio using randomeffects models. Heterogeneity was assessed using the $I^{2}$ test, and $I^{2}$ of $>50 \%$ was considered a moderate interstudy variation. A $p$ value $<0.05$ was considered statistically significant. All analyses were performed using the OpenMetaAnalyst software.

\section{Results}

The flow chart depicting the process of study screening is presented in Fig. 1. An initial electronic search yielded 65 articles, and 3 others were identified via cross-reference search. After excluding duplicates, 41 studies were found to be eligible for screening their abstracts and full texts. Out of the 41 screened, 23 were not relevant to our study, 5 were review articles/editorials, and the rest did not contain extractable data. Eight studies were included in the quantitative analysis of the pooled odds ratio (OR). The total sample size consisted of 8142 COVID-positive patients, out of which 833 patients had NAFLD. The characteristics of the included studies are summarized in Table 1.

The pooled OR for severe COVID-19 in NAFLD, after adjusting for obesity was 2.358 (95\% CI: 1.902-2.923, $p<0.001$; Fig. 2). A minimal heterogeneity was identified in the analysis. No significant differences were observed in the leave-one-out sensitivity analysis for disease severity.

\section{Discussion}

Patients with pre-existing liver disorders, such as liver cirrhosis and hepatocellular carcinoma, are considered to have a higher susceptibility for any kind of infection and sepsis secondary to impaired host defense [11]. Cardiometabolic risk factors such as obesity also have been identified as potential risk factors for mortality in COVID-19 [12].

Table 1 Baseline characteristics of included studies

\begin{tabular}{|c|c|c|c|c|c|}
\hline Study & Location & $\begin{array}{l}\text { Sample } \\
\text { size }\end{array}$ & Males & $\begin{array}{l}\text { Patients with } \\
\text { NAFLD }\end{array}$ & Patient outcome for the severity of disease (COVID-19) included in our analysis \\
\hline $\begin{array}{c}\text { Mahamid } \\
{[3]}\end{array}$ & Israel & 71 & 20 & 22 & $\begin{array}{l}\text { COVID-19 severity assessed using diagnosis and treatment protocol for novel Coronavirus } \\
\text { pneumonia released by National Health Commission and State Administration of } \\
\text { Traditional Chinese Medicine* }\end{array}$ \\
\hline Targher [4] & China & 310 & 149 & 94 & Criteria for severe COVID-19 a laid down by the National Health Commission, China \\
\hline $\begin{array}{c}\text { Zhou et al. } \\
1[5]\end{array}$ & China & 327 & - & 93 & Criteria for severe COVID-19 a laid down by the National Health Commission, China \\
\hline $\begin{array}{l}\text { Zho et al. } 2 \\
{[6]}\end{array}$ & China & 110 & 82 & 55 & Criteria for severe COVID-19 a laid down by the National Health Commission, China \\
\hline Ji et al. [7] & China & 202 & 113 & 76 & According to Chinese National Health commission \\
\hline $\begin{array}{c}\text { Gao et al. } \\
{[8]}\end{array}$ & China & 130 & 82 & 65 & Criteria for severe COVID-19 a laid down by the National Health Commission, China \\
\hline $\begin{array}{l}\text { Hashemi } \\
\quad \text { et al. [9] }\end{array}$ & USA & 363 & 201 & 55 & ICU admission \\
\hline $\begin{array}{l}\text { Bramante } \\
\quad \text { et al. [10] }\end{array}$ & USA & 6700 & 3752 & 373 & Hospitalization \\
\hline
\end{tabular}

*National Health Commission; National Administration of Traditional Chinese Medicine. Diagnosis and treatment protocol for novel coronavirus pneumonia (Trial Version 7). Chin Med J (Engl) 2020; 133:1087-1095 
Fig. 1 Study flow chart

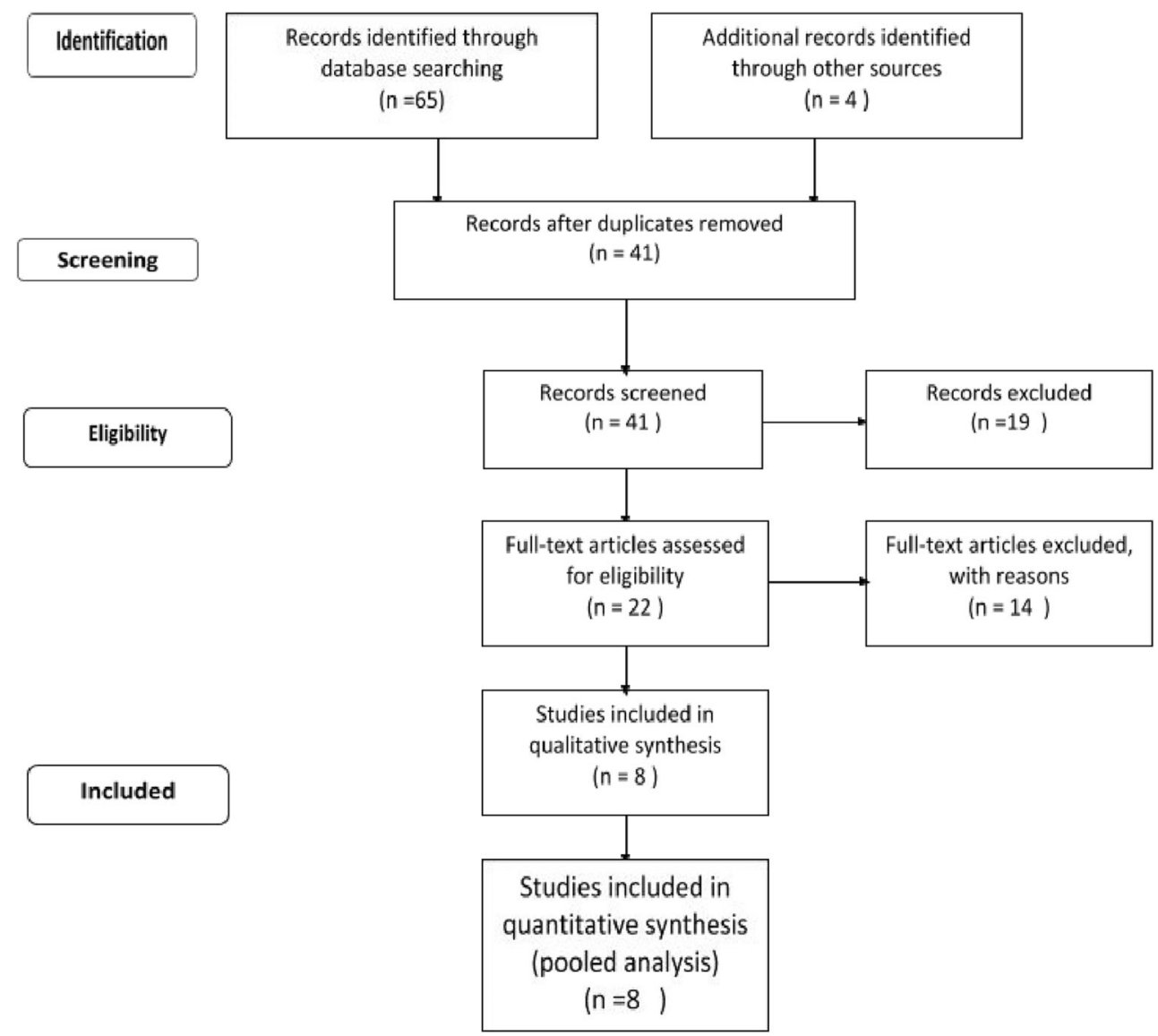

Nonalcoholic fatty liver disease, NAFLD (also known as metabolic associated fatty liver disease, MAFLD), is considered to be the hepatic manifestation of metabolic syndrome, which encompasses obesity, diabetes, dyslipidemia, and insulin resistance [13]. It is understandable that due to these concurrent issues, NAFLD would predispose to severe COVID19. A multicenter retrospective study by Zheng et al. further validated this information. They showed that obesity conferred $\mathrm{a} \sim$ sixfold higher risk of severe COVID-19 in patients with NAFLD. The OR for the severity of COVID-19 illness was 6.32 in obese NAFLD patients than in non-obese patients with NAFLD [14].

However, our pooled analysis points out yet another interesting observation about COVID-19 and fatty liver disease. We found that NAFLD was associated with an increased risk of severe COVID-19, even after adjusting for obesity as a possible confounding factor. We believe that liver fat and associated inflammation could exacerbate the virusassociated cytokine storm, leading to worsening COVID-19. Targher et al. found that increasing liver fibrosis measured by

\begin{tabular}{|c|c|}
\hline Studies & Estimate (95\% C.I.) \\
\hline Gao et al & $4.070(1.100,15.059)$ \\
\hline Ji et al & $6.400(1.313,31.200)$ \\
\hline Zhou et al 1 & $2.670(1.130,6.309)$ \\
\hline Mahamid et al & $3.570(1.220,10.447)$ \\
\hline Zhou et al 2 & $4.070(1.200,13.804)$ \\
\hline Bramante et al & $2.040(1.550,2.685)$ \\
\hline Targher et al & $2.950 \quad(1.370$ \\
\hline Hashemi et al & $2.300 \quad(1.270$ \\
\hline Overall $\left(I^{\wedge} 2=0 \%, P=0.658\right)$ & $2.358(1.902$, \\
\hline
\end{tabular}

Fig. 2 Pooled odds ratio for severe COVID-19




NFS/FIB-4 score was linked to increased severity of disease in COVID-19 patients [4]. Moreover, liver fat has been independently linked to an increased risk of testing positive for COVID-19 [15]. A study showed that NAFLD was independently associated with a significant ALT elevation after adjusting for BMI and other factors [16].

Hence, it is conceivable that injury caused by fat accumulation in the liver and subsequent fibrosis could be one of the plausible reasons for the poor disease course observed in patients with NAFLD.

\section{Conclusions}

Nonalcoholic fatty liver disease is associated with a higher risk of symptomatic, severe, and progressive COVID-19. The association is significant even after adjusting for an important confounding factor, obesity. Hence, it can be concluded that the hepatic manifestation of COVID-19 is independently linked to the severity of coronavirus disease. However, the exact relationship between liver fat and COVID-19 remains to be elucidated.

Author Contributions Conception and design: Sonali Sachdeva and Harshwardhan Khandait

Literature search: Sonali Sachdeva, Harshwardhan Khandait, and Rupak Desai

First draft: Sonali Sachdeva and Jonathan Kopel

Critical revision and editing: All authors

Final approval: All authors

All authors have made substantive contributions to the study, and all authors endorse the data and conclusions.

\section{References}

1. Boregowda U, Aloysius MM, Perisetti A, Gajendran M, Bansal P, Goyal H. Serum activity of liver enzymes is associated with higher mortality in COVID-19: a systematic review and meta-analysis. Front Med (Lausanne). 2020;7:431.

2. Medeiros AK, Barbisan CC, Cruz IR, de Araújo EM, Libânio BB, Albuquerque KS, et al. Higher frequency of hepatic steatosis at CT among COVID-19-positive patients. Abdom Radiol (NY). 2020;45(9):2748-54. https://doi.org/10.1007/s00261-020-026487.

3. Mahamid M, Nseir W, Khoury T, Mahamid B, Nubania A, SubLaban K, et al. Nonalcoholic fatty liver disease is associated with COVID-19 severity independently of metabolic syndrome: a retrospective case-control study. Eur J Gastroenterol Hepatol. 2020. https://doi.org/10.1097/MEG.0000000000001902 Publish Ahead of Print.

4. Targher G, Mantovani A, Byrne CD, Wang XB, Yan HD, Sun QF, et al. Risk of severe illness from COVID-19 in patients with metabolic dysfunction-associated fatty liver disease and increased fibrosis scores. Gut. 2020;69(8):1545-7. https://doi.org/10.1136/ gutjnl-2020-321611 Epub 2020 May 15.

5. Zhou YJ, Zheng KI, Wang XB, Yan HD, Sun QF, Pan KH, et al. Younger patients with MAFLD are at increased risk of severe COVID-19 illness: a multicenter preliminary analysis. J Hepatol. 2020;73(3):719-21. https://doi.org/10.1016/j.jhep.2020.04.027.

6. Zhou YJ, Zheng KI, Wang XB, Sun QF, Pan KH, Wang TY, et al. Metabolic-associated fatty liver disease is associated with severity of COVID-19. Liver Int. 2020;40(9):2160-3. https://doi.org/10. 1111/liv.14575.

7. Ji D, Qin E, Xu J, Zhang D, Cheng G, Wang Y, et al. Non-alcoholic fatty liver diseases in patients with COVID-19: A retrospective study. J Hepatol. 2020;73(2):451-3. https://doi.org/10.1016/j.jhep. 2020.03.044.

8. Gao F, Zheng KI, Wang XB, Yan HD, Sun QF, Pan KH, et al. Metabolic associated fatty liver disease increases coronavirus disease 2019 disease severity in nondiabetic patients. J Gastroenterol Hepatol. 2020. https://doi.org/10.1111/jgh.15112.

9. Hashemi N, Viveiros K, Redd WD, Zhou JC, McCarty TR, Bazarbashi AN, et al. Impact of chronic liver disease on outcomes of hospitalized patients with COVID-19: a multicentre United States experience. Liver Int. 2020. https://doi.org/10.1111/liv. 14583.

10. Bramante C, Tignanelli CJ, Dutta N, Jones E, Tamariz L, Clark JM, Usher M, Metlon-Meaux G, Ikramuddin S. Non-alcoholic fatty liver disease (NAFLD) and risk of hospitalization for Covid-19. medRxiv [Preprint]. 2020:2020.09.01.20185850. https://doi.org/ 10.1101/2020.09.01.20185850.

11. Strnad P, Tacke F, Koch A, Trautwein C. Liver - guardian, modifier and target of sepsis. Nat Rev Gastroenterol Hepatol. 2017;14(1):55-66.

12. Hussain A, Mahawar K, Xia Z, Yang W, El-Hasani S. Obesity and mortality of COVID-19. Meta-analysis. Obes Res Clin Pract. 2020;14(4):295-300. https://doi.org/10.1016/j.orcp.2020.07.002.

13. Hashimoto E, Taniai M, Tokushige K. Characteristics and diagnosis of NAFLD/NASH. J Gastroenterol Hepatol. 2013;28(Suppl 4): 64-70. https://doi.org/10.1111/jgh.12271.

14. Zheng KI, Gao F, Wang XB, Sun QF, Pan KH, Wang TY, et al. Letter to the Editor: Obesity as a risk factor for greater severity of COVID-19 in patients with metabolic associated fatty liver disease. Metabolism. 2020;108:154244. https://doi.org/10.1016/j.metabol. 2020.154244.

15. Roca-Fernández A, Dennis A, Nicolls R, McGonigle J, Kelly M, Banerjee R. High liver fat associates with higher risk of developing symptomatic COVID-19 infection - initial UK biobank observations. 2020. https://doi.org/10.1101/2020.06.04.20122457.

16. Huang R, Zhu L, Wang J, Xue L, Liu L, Yan X, et al. Clinical features of COVID-19 patients with non-alcoholic fatty liver disease. Hepatol Commun. 2020. https://doi.org/10.1002/hep4.1592.

This manuscript, as submitted or its essence in another version, is not under consideration for publication elsewhere and will not be published elsewhere while under review by Digestive and Liver Diseases.

Publisher's Note Springer Nature remains neutral with regard to jurisdictional claims in published maps and institutional affiliations. 